\title{
Changing Demographics of Stroke Mimics in Present Day Stroke Code Era: Need of a Streamlined Clinical Assessment for Emergency Physicians
}

\author{
Kaushik Sundar ${ }^{1}$ Ajay Panwar ${ }^{2}$ Lomesh Bhirud ${ }^{3}$ Eldho Mathew Paul ${ }^{4}$ Paul J. Alapatt $^{5}$ \\ Gigy Varkey Kuruttukulam ${ }^{5}$
}

${ }^{1}$ Department of Neurology, Rabindranath International Institute of Cardiac Sciences, Kolkata, West Bengal, India

${ }^{2}$ Department of Neurology, Rotary Ambala Cancer and General Hospital, Ambala, Haryana, India

${ }^{3}$ Department of Neurology, Kiran Hospital, Surat, Gujrat, India

${ }^{4}$ Department of Pharmacology, Rajagiri Hospital, Kochi, India

${ }^{5}$ Department of Neurology, Rajagiri Hospital, Kochi, India

\begin{abstract}
Address for correspondence Ajay Panwar, MD, DM, Department of Neurology, Rotary Ambala Cancer and General Hospital,

Ambala 133001, Haryana, India (e-mail: ajay19panwar@gmail.com).
\end{abstract}

J Neurosci Rural Pract 2021;12:550-554.

\begin{abstract}
Background There is an apparently high incidence of stroke mimics in the present-day stroke code era. The reason being is the intense pressure to run with time to achieve the "time is brain"-based goals.

Methods The present study was a retrospective analysis of the data collected over a duration of 6 months from April 2019 to September 2019. We observed the incidence of stroke mimics among the patients for whom rapid response stroke code was activated during the study period. We also performed a logistic regression analysis to identify the clinical features which can act as strong predictors of stroke and mimics.

Results A total of 314 stroke codes were activated of which 256 (81.5\%) were stroke and $58(18.5 \%)$ were the mimics. Functional disorders and epilepsy were the most common mimics (24.1\% each). Female gender ( $p=0.04$; odds ratio [OR] 2.9[1.0-8.8]), isolated impairment of consciousness ( $p<0.01$; OR 4.3[1.5-12.6]), and isolated dysarthria

Keywords

- stroke

- stroke mimic

- stroke code

- predictors $(p<0.001)$ were the strong independent predictors for a stroke mimic. Hemiparesis was the strong independent predictor for a stroke ( $p<0.001$; OR $0.0[0.0-0.1]$ ).

Conclusion In the present epoch of rapid response stroke management, a streamlined assessment by the emergency physicians based on the above clinical predictors may help in avoiding the misdiagnosis of a mimic as stroke.
\end{abstract}

\section{Introduction}

The modern stroke era has revolutionized the management of acute ischemic stroke. The functional outcome of ischemic stroke patients has greatly improved with the advent of intravenous (IV) thrombolysis with recombinant tissue plasminogen activator (rt-PA) and endovascular procedures for thrombus retrieval. ${ }^{1,2}$ The successful outcome of IV and intra-arterial therapy is dependent on the time window and the benefit decreases as the time of treatment initiation after

published online June 16, 2021
DOI https://doi.org/

10.1055/s-0041-1727419

ISSN 0976-3147 the stroke onset increases. ${ }^{3}$ To realize the notion of "time is brain" in stroke management, a rapid-response stroke code (SC) system is crucial and is an integral part of most present-day comprehensive stroke centers. ${ }^{4}$ The emergency department (ED) is the initial link of an in-hospital SC chain; and hence, plays a crucial role in the smooth functioning of an SC system. ${ }^{5}$ However, the promptness to achieve the time-based goals in the present era of stroke management often results in SC activations in several conditions mimicking stroke.

(c) 2021. Association for Helping Neurosurgical Sick People.

This is an open access article published by Thieme under the terms of the Creative Commons Attribution-NonDerivative-NonCommercial-License, permitting copying and reproduction so long as the original work is given appropriate credit. Contents may not be used for commercial purposes, or adapted, remixed, transformed or built upon. (https://creativecommons.org/licenses/by-nc-nd/4.0/).

Thieme Medical and Scientific Publishers Pvt. Ltd. A-12, 2nd Floor, Sector 2, Noida-201301 UP, India 
Consequently, there is an apparent rise in the frequency of stroke mimics (SMs) in this epoch of stroke treatment.

We planned to analyze the incidence of SM and the spectrum of conditions mimicking stroke among the SC activations in our ED. We also aimed to identify the clinical features which would help to reliably distinguish a stroke from the SM in the ED.

\section{Methods}

The present study was a retrospective analysis of the data collected over a duration of 6 months from April 2019 to September 2019. It was conducted at Rajagiri hospital, Kochi which is a high-volume center for IV thrombolysis and endovascular stroke procedures. The study was approved by the Institutional Ethics Committee.

\section{Inclusion and Exclusion Criteria}

The patients for whom SC was activated during the study duration were included in the study if $(1)$ they aged $\geq 18$ years;
(2) Magnetic resonance imaging (MRI) of the brain was done for ischemic stroke evaluation provided that the computed tomographic (CT) scan was negative for hemorrhage.

The patients were classified to have a stroke only if they had a "definite stroke," i.e., the clinical picture of an acute vascular event was supported by the neuroimaging evidence. ${ }^{6}$ Those diagnosed as a transient ischemic attack (TIA) were excluded from the study. Subarachnoid hemorrhage and subdural hemorrhage as well, were not considered stroke for the purpose of our study and hence excluded. ${ }^{7}$

\section{Diagnosis and Treatment Protocol}

Initially, all the patients presenting to the ED were assessed by an emergency physician, who then activated SC for the suspected stroke cases. All the SC activated patients were initially seen by a stroke fellow and subsequently, by a senior stroke neurologist. A stroke fellow in our setting was a certified neurologist registered with a 3-year degree course of "Doctorate of Medicine" in neurology, currently undergoing a fellowship in stroke and neurointervention. The details of

Table 1 Basic characteristics of the patients with stroke and its mimics

\begin{tabular}{|c|c|c|c|c|}
\hline & $\begin{array}{l}\text { Total stroke code } \\
\text { activations }=314\end{array}$ & $\begin{array}{l}\text { Stroke } \\
N=256\end{array}$ & $\begin{array}{l}\text { Stroke mimics } \\
N=58\end{array}$ & $p$-Value \\
\hline Age in years (Mean \pm SD) & $61.4 \pm 13.1$ & $62.1 \pm 13.1$ & $58.9 \pm 12.8$ & 0.10 \\
\hline Gender & $\begin{array}{l}\mathrm{M}=188(59.9 \%) \\
\mathrm{F}=126(40.1 \%)\end{array}$ & $\begin{array}{l}M=161(62.9 \%) \\
F=95(37.1 \%)\end{array}$ & $\begin{array}{l}\mathrm{M}=27(46.6 \%) \\
\mathrm{F}=31(53.4 \%)\end{array}$ & 0.02 \\
\hline \multicolumn{5}{|l|}{ Clinical features } \\
\hline Hemiparesis & $207(65.9 \%)$ & $202(78.9 \%)$ & $5(8.6 \%)$ & $<0.001$ \\
\hline Isolated impaired consciousness & $78(24.8 \%)$ & $44(17.1 \%)$ & $34(58.6 \%)$ & $<0.001$ \\
\hline Headache & $25(07.9 \%)$ & $19(7.5 \%)$ & $06(10.3 \%)$ & 0.47 \\
\hline Vertigo & $62(19.7 \%)$ & $45(17.6 \%)$ & $17(29.3 \%)$ & 0.04 \\
\hline Isolated vertigo & 01 (00.3\%) & $00(00.0 \%)$ & 01 (01.7\%) & 0.03 \\
\hline Seizure & $17(05.4 \%)$ & $10(03.9 \%)$ & $07(12.1 \%)$ & 0.01 \\
\hline Monoparesis & $21(06.7 \%)$ & $17(06.6 \%)$ & $04(06.9 \%)$ & 0.95 \\
\hline Paraparesis & $04(01.3 \%)$ & $00(00.0 \%)$ & $04(06.9 \%)$ & $<0.001$ \\
\hline Quadriparesis & $08(02.5 \%)$ & $06(02.3 \%)$ & $02(03.4 \%)$ & 0.64 \\
\hline Dysarthria & $155(49.3 \%)$ & $136(53.1 \%)$ & $19(32.8 \%)$ & $<0.001$ \\
\hline Isolated dysarthria & $16(05.1 \%)$ & $01(00.4 \%)$ & $15(25.9 \%)$ & $<0.001$ \\
\hline Gaze preference & $50(15.9 \%)$ & $48(18.7 \%)$ & $02(03.4 \%)$ & $<0.001$ \\
\hline Hemianopia & $10(03.2 \%)$ & $08(03.1 \%)$ & $02(03.4 \%)$ & 0.91 \\
\hline Facial palsy & $165(52.5 \%)$ & $148(57.8 \%)$ & $17(29.3 \%)$ & $<0.001$ \\
\hline Aphasia & $75(23.9 \%)$ & $67(26.2 \%)$ & $08(13.8 \%)$ & 0.04 \\
\hline Hemisensory involvement & $42(13.4 \%)$ & $33(12.9 \%)$ & $09(15.5 \%)$ & 0.61 \\
\hline $\begin{array}{l}\text { Isolated hemisensory symptoms with } \\
\text { no objective deficit }\end{array}$ & $05(01.6 \%)$ & $02(00.8 \%)$ & $03(05.2 \%)$ & 0.01 \\
\hline Ataxia & $71(22.6 \%)$ & $60(23.4 \%)$ & $11(19.0 \%)$ & 0.45 \\
\hline \multicolumn{5}{|l|}{ Risk factors } \\
\hline Hypertension & $220(70.1 \%)$ & $193(75.4 \%)$ & $27(46.6 \%)$ & $<0.001$ \\
\hline Diabetes & 115 (36.4\%) & $100(39.1 \%)$ & 15 (25.9\%) & 0.06 \\
\hline Atrial fibrillation & $12(03.8 \%)$ & $10(03.9 \%)$ & $02(03.4 \%)$ & 0.87 \\
\hline Ischemic heart disease & $40(12.7 \%)$ & $30(11.7 \%)$ & $10(17.2 \%)$ & 0.25 \\
\hline
\end{tabular}

Note: Bold values indicate significant p-values. All values $\leq 0.05$ were taken as significant. 
the initial assessment of the patient were recorded by the fellow on structured data forms. The patient's history regarding the presence of vascular risk factors and history of stroke or TIA were recorded. The details of the factors associated with SM as cognitive dysfunction, seizures, migraine, and psychological impairment were also noted. Based on the consensus clinical diagnosis, the patients with a strong suggestion of stroke were treated by stroke thrombolysis after ruling out a cerebral hemorrhage on CT brain. Those with a less convincing possibility of stroke underwent diffusion weighted (DW), apparent diffusion coefficient, and gradient echo sequences of MRI. Of these cases, those diagnosed as acute ischemic stroke were treated with IV thrombolysis, if the clinical criteria for thrombolysis were met. ${ }^{8}$ In every stroke case, we undertook one noninvasive angiographic study of cerebral and neck vessels, either magnetic resonance angiography or CT angiography. Those having a large vessel occlusion including internal carotid artery (ICA), ICA terminus (T occlusion), middle cerebral artery-M1, or basilar artery were immediately shifted to the catheterization laboratory (cath lab) for endovascular intervention. Informed risk consent was taken from the patient's close relatives for stroke thrombolysis as well as endovascular interventions.

An SM was diagnosed when the clinical picture was not typical for a vascular event and an alternative etiology was found which could reliably explain the symptoms.

\section{Data Retrieval}

The clinical and neuroimaging details of all the patients for whom SC is activated are routinely entered into the central data repository of our hospital. We conducted a retrospective data retrieval of the prospectively entered data according to the above-defined inclusion criteria.

\section{Statistical Analysis}

Statistical analysis was performed using IBM.SPSS version 23.0 (SPSS Inc., Chicago, Illinois, United States). We computed categorical variables as percentages and continuous variables as mean \pm S.D. Categorical variables were compared using Chi-square test. All $p$-values less than 0.01 were regarded as significant. The demographic variables, clinical features, and risk factors were tested on a binomial logistic regression model using univariate and multivariate analysis, for observing the predictors of a SM. $p$-Value, odds ratio (OR), and confidence interval were recorded for the various predictors.

\section{Results}

A total of 314 SCs were activated during the study duration. Of these, 188 (60\%) were male patients and 126 (40\%) were female. The number of stroke cases was 256 (81.5\%) and the SM was 58 (18.5\%). Basic characteristics of the patients among the study groups are shown in - Table 1.

\section{Frequency distribution of stroke mimics}

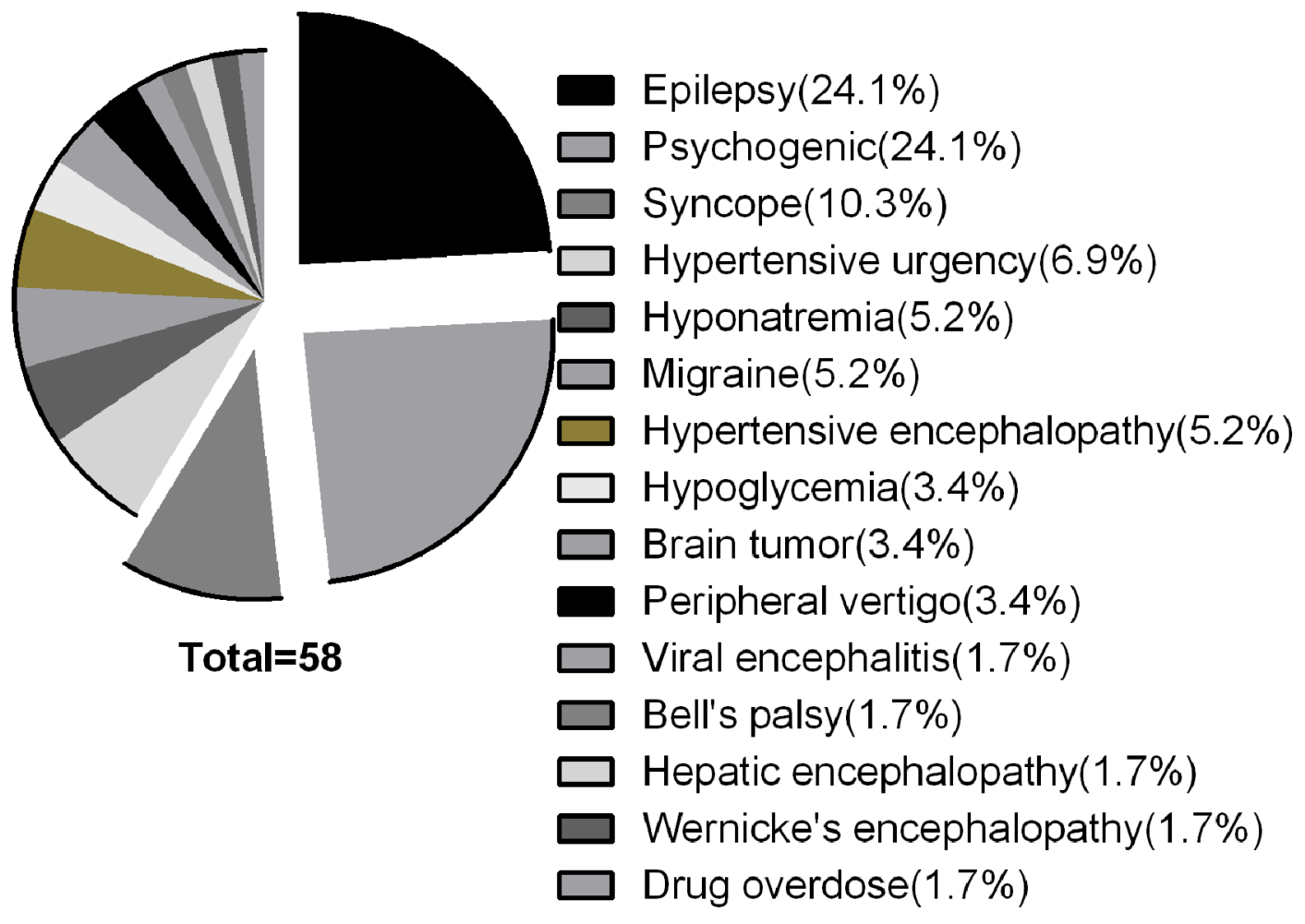

Fig. 1 A graphical representation of the frequency distribution of stroke mimics. 


\section{Frequency Distribution of the Stroke Mimics}

The most common etiology of SM in our study were functional/psychological and epilepsy (24.1\% each). Besides these, another condition which frequently mimicked stroke was syncope (10.3\%). - Fig. 1 shows the frequency distribution of various SMs in our study.

\section{Predictors of Stroke and the Mimics}

On univariate regression analysis, the factors which were predictors of SM were female gender, seizure, vertigo, isolated dysarthria, isolated impairment of consciousness without any focal neurological signs, and isolated hemisensory symptoms with no objective sensory loss. Of these, isolated impairment of consciousness and isolated subjective hemisensory involvement strongly $(p<0.001)$ predicted an SM. The factors which were predictors of stroke were hemiparesis, dysarthria, facial palsy, aphasia, and hypertension. Of these, facial palsy, hemiparesis, and hypertension strongly $(p<0.001)$ predicted a stroke. Hemiparesis had the highest odds (OR 0.0[0.0-0.1]) against a SM (- Table 2). Dysarthria, when accompanied with other neurological deficits was a predictor of stroke; however, when present in isolation with no other deficit predicted a mimic.

On univariate analysis, variables which had a significant association with stroke and SM were entered into a multivariate analysis model. On multivariate regression analysis, the factors which were found to be the strong independent predictors of a SM were female gender, isolated dysarthria, and isolated impairment of consciousness. Hemiparesis was the sole independent predictor of stroke, with very high odds against an SM (OR 0.0[0.0-0.1]).

\section{Discussion}

In the present study, $18.5 \%$ of the patients for whom SC was activated were identified as SM. This high frequency of SM may be attributed to intense pressure to achieve time-based targets, viz. door-to-needle times in acute stroke settings. ${ }^{9}$ More interestingly, the spectrum of the conditions mimicking the stroke has changed in the SC era. Conventionally, conversion disorder or functional disorders were reported as the least frequently occurring mimics. ${ }^{10}$ On the contrary, we observed them as the most common condition mimicking stroke alongside epilepsy. In the haste to run with time, it often becomes impractical to perform a detailed neurological examination in an SC-activated patient, and thus, to rule out an organic disease clinically. This changing trend of SM was also reflected in a recently conducted large-scale study by Wilkins et al in which they observed $8.2 \%$ of the SC patients as functional mimics. ${ }^{11}$ Hypoglycemia has been classically known as an SM; however, with routinely used point-of-care testing nowadays, it is often ruled out before activating SC. ${ }^{12,13}$ Consequently, it is being less frequently seen as an SM, as in our study. So, with the advent of SC era, the demographics of SM have significantly changed.

In the study by Wilkins et al, $9.2 \%$ of the functional mimics received stroke thrombolysis. ${ }^{11}$ In another study by Goyal et al,
Table 2 Logistic regression analysis of various predictors of the stroke and the mimics

\begin{tabular}{|c|c|c|}
\hline & $p$-Value & $\mathrm{OR}(\mathrm{Cl})$ \\
\hline \multicolumn{3}{|l|}{ Univariate analysis } \\
\hline Gender & 0.02 & $1.9(1.0-3.4)$ \\
\hline \multicolumn{3}{|l|}{ Clinical features } \\
\hline $\begin{array}{l}\text { Isolated impaired } \\
\text { consciousness }\end{array}$ & $<0.001$ & $6.8(3.6-12.6)$ \\
\hline Headache & 0.47 & $1.4(0.5-3.7)$ \\
\hline Vertigo & 0.04 & $1.9(1.0-3.7)$ \\
\hline Isolated vertigo & 1.00 & NA \\
\hline $\begin{array}{l}\text { Isolated subjective hemi- } \\
\text { sensory involvement }\end{array}$ & 0.03 & $7.0(1.1-43.2)$ \\
\hline Seizure & 0.02 & $3.3(1.2-9.2)$ \\
\hline Monoparesis & 0.95 & $1.0(0.3-3.2)$ \\
\hline Paraparesis & 0.99 & NA \\
\hline Quadriparesis & 0.64 & $1.4(0.2-7.5)$ \\
\hline Isolated dysarthria & $<0.001$ & $\begin{array}{l}88.9 \\
(11.4-690.8)\end{array}$ \\
\hline Dysarthria & $<0.01$ & $0.4(0.2-0.7)$ \\
\hline Gaze preference & 0.01 & $0.1(0.0-0.6)$ \\
\hline Hemianopia & 0.91 & $1.0(0.2-5.2)$ \\
\hline Facial palsy & $<0.001$ & $0.2(0.1-0.5)$ \\
\hline Aphasia & 0.04 & $0.4(0.2-0.9)$ \\
\hline Hemisensory involvement & 0.55 & $1.2(0.5-2.8)$ \\
\hline Ataxia & 0.44 & $0.7(0.3-1.5)$ \\
\hline Hemiparesis & $<0.001$ & $0.0(0.0-0.1)$ \\
\hline \multicolumn{3}{|l|}{ Risk factors } \\
\hline Hypertension & $<0.001$ & $0.2(0.1-0.5)$ \\
\hline Diabetes & 0.06 & $0.5(0.2-1.0)$ \\
\hline Atrial fibrillation & 0.87 & $0.8(0.1-4.1)$ \\
\hline Ischemic heart disease & 0.25 & $1.5(0.7-3.4)$ \\
\hline \multicolumn{3}{|l|}{ Multivariate analysis } \\
\hline Female gender & 0.04 & $2.9(1.0-8.8)$ \\
\hline $\begin{array}{l}\text { Isolated impaired } \\
\text { consciousness }\end{array}$ & $<0.01$ & $4.3(1.5-12.6)$ \\
\hline Seizure & 0.26 & $2.8(0.4-18.2)$ \\
\hline Isolated dysarthria & $<0.001$ & NA \\
\hline Dysarthria & 0.09 & $0.3(0.1-1.2)$ \\
\hline Hemiparesis & $<0.001$ & $0.0(0.0-0.1)$ \\
\hline Gaze preference & 0.47 & $0.4(0.0-4.7)$ \\
\hline Facial palsy & 0.07 & $0.3(0.1-1.1)$ \\
\hline Aphasia & 0.52 & $0.6(0.1-2.6)$ \\
\hline Hypertension & 0.11 & $0.4(0.1-1.2)$ \\
\hline Vertigo & 0.38 & $1.6(0.5-5.2)$ \\
\hline $\begin{array}{l}\text { Isolated subjective } \\
\text { hemisensory involvement }\end{array}$ & 0.59 & $1.8(0.2-15.8)$ \\
\hline
\end{tabular}

Abbreviations: $\mathrm{Cl}$, confidence interval; NA, not applicable; OR, odds ratio

Note: Bold values indicate significant $p$-values. All values $\leq 0.05$ were taken as significant. 
it was observed that treating SMs with IV rt-PA resulted in excess direct and indirect costs of $\$ 257,975$ and $\$ 152,813$, respectively, to the hospital. ${ }^{14}$ So, to avoid the undue bleeding risk as well as the costs associated with unwarranted thrombolysis, it becomes imperative to reliably distinguish stroke from SM based on the clinical assessment in ED. Thus, we performed a rigorous logistic regression analysis to identify the clinical predictors of stroke and SM. We observed female gender, isolated impairment in consciousness, and isolated dysarthria as the strong independent predictors of a mimic. On the other hand, hemiparesis was the only strong independent predictor of a stroke case. Based on these predictors, a streamlined clinical examination by the emergency physicians may help in avoiding misdiagnosis. The signs which help in differentiating organic from nonorganic deficits should be focused upon, in patients based on the clinical predictors. ${ }^{15,16}$ Further, patients suspected to be SM based on these predictors should not undergo stroke thrombolysis just after ruling out a hemorrhage on CT scan. A DW sequence of MRI must be a part of stroke protocol in such cases; rather, it would be ideal if a single DW-MRI sequence is incorporated in the routine SC pathway. With that said, a single sequence MRI for the SC patient at our hospital does not cause a delay of more than 5 minutes. We consider trading of these few minutes for the diagnostic accuracy and preventing unjust stroke thrombolysis, as worthwhile.

Our study was a retrospective study, and it would be ideal to observe the clinical predictors of SMs in a prospective large-scale study. Notwithstanding the same, this study will possibly serve as a reference for future studies in this regard.

\section{Conflict of Interest}

None declared.

\section{Funding}

None.

\section{References}

1 Hacke W, Donnan G, Fieschi C, et al. ATLANTIS Trials InvestigatorsECASS Trials InvestigatorsNINDS rt-PA Study Group Investigators. Association of outcome with early stroke treatment: pooled analysis of ATLANTIS, ECASS, and NINDS rt-PA stroke trials. Lancet 2004;363(9411) :768-774
2 Nogueira RG, Liebeskind DS, Sung G, Duckwiler G, Smith WS; MERCI. Multi MERCI Writing Committee. Predictors of good clinical outcomes, mortality, and successful revascularization in patients with acute ischemic stroke undergoing thrombectomy: pooled analysis of the Mechanical Embolus Removal in Cerebral Ischemia (MERCI) and Multi MERCI Trials. Stroke 2009;40(12):3777-3783

3 Muchada M, Rodriguez-Luna D, Pagola J, et al. Impact of time to treatment on tissue-type plasminogen activator-induced recanalization in acute ischemic stroke. Stroke 2014;45(9):2734-2738

4 Saver JL. Time is brain-quantified. Stroke 2006;37(1):263-266

5 El Husseini N, Goldstein LB. "Code stroke": hospitalized versus emergency department patients. J Stroke Cerebrovasc Dis 2013;22(4):345-348

6 Natteru P, Mohebbi MR, George P, Wisco D, Gebel J, Newey CR. Variables that best differentiate in-patient acute stroke from stroke-mimics with acute neurological deficits. Stroke Res Treat 2016;2016:4393127

7 Hand PJ, Kwan J, Lindley RI, Dennis MS, Wardlaw JM. Distinguishing between stroke and mimic at the bedside: the brain attack study. Stroke 2006;37(3):769-775

8 Powers WJ, Rabinstein AA, Ackerson T, et al. American Heart Association Stroke Council. 2018 guidelines for the early management of patients with acute ischemic stroke: a guideline for healthcare professionals from the American Heart Association/American Stroke Association. Stroke 2018;49(3):e46-e110

9 Tan BYQ Ngiam NJH, Sunny S, et al. Improvement in door-to-needle time in patients with acute ischemic stroke via a simple stroke activation protocol. J Stroke Cerebrovasc Dis 2018;27(6):1539-1545

10 Libman RB, Wirkowski E, Alvir J, Rao TH. Conditions that mimic stroke in the emergency department. Implications for acute stroke trials. Arch Neurol 1995;52(11):1119-1122

11 Wilkins SS, Bourke P, Salam A, et al. Functional stroke mimics: incidence and characteristics at a primary stroke center in the Middle East. Psychosom Med 2018;80(5):416-421

12 Berkovic SF, Bladin PF, Darby DG. Metabolic disorders presenting as stroke. Med J Aust 1984;140(7):421-424

13 Shotliff K, Prasad A, Millard P. Hypoglycaemia masquerading as a stroke. Postgrad Med J 1992;68(804):843

14 Goyal N, Male S, Al Wafai A, Bellamkonda S, Zand R. Cost burden of stroke mimics and transient ischemic attack after intravenous tissue plasminogen activator treatment. J Stroke Cerebrovasc Dis 2015;24(4):828-833

15 Mendez MF. Non-neurogenic language disorders: a preliminary classification. Psychosomatics 2018;59(1):28-35

16 Sonoo M. Abductor sign: a reliable new sign to detect unilateral non-organic paresis of the lower limb. J Neurol Neurosurg Psychiatry 2004;75(1):121-125 